\title{
REDUCCIÓN FENOMENOLÓGICA Y FIGURAS DE LA EXCEDENCIA
}

\section{Roberto J. Walton *}

En este trabajo se consideran figuras de la excedencia desarrolladas en un tercer estadio de la fenomenología que se presenta a sí mismo como más originario que los niveles desplegados por Husserl y Heidegger. La fenomenología material de Michel Henry se ocupa de una fenomenalidad previa en la pura inmanencia de la autoexperiencia. La fenomenología estructural de Heinrich Rombach pone de relieve como fenómeno fundamental un avance creador en que el advenimiento de un mundo nuevo implica un "más" respecto del mundo dado. La fenomenología de la donación de Jean-Luc Marion describe el fenómeno saturado que sobrepasa toda posible determinación conceptual gracias a la sobreabundancia de la intuición en relación con el mundo anticipable. Las tres formas de fenomenología -ligadas respectivamente a un análisis de la originariedad, el estallido y la saturación del fenómeno- serán examinadas de acuerdo con el modo en que la excedencia destacada se conecta con la correlación entre lo que aparece y su aparecer, es decir, el tema central de la fenomenología de Husserl.

\section{La fenomenalidad originaria}

Henry afirma que el tema de la fenomenología es la esencia de la manifestación, es decir, el fundamento o condición de posibilidad en virtud del cual algo puede manifestarse. Según Husserl, esa condición se encuentra en la intencionalidad. El darse del objeto supone su correlación con las operaciones de una conciencia, y este colocar delante en la condición de objeto implica una puesta a distancia, es decir, la separación intencional entre el yo y su correlato. Según Heidegger, la esencia de la manifestación es el movimiento de trascendencia que va más allá de los entes en dirección a su ser. $\mathrm{Y}$ esta instauración de un medio de apertura significa también una

* Doctor en Filosofía. Investigador Superior del CONICET. Profesor en la Universidad de Buenos Aires y en la Universidad Católica de Santa Fe. 
distancia entre el Dasein y los entes ${ }^{1}$. En contraste con estas posiciones, Henry sostiene que la manifestación de los fenómenos trascendentes remite a una manifestación originaria en virtud de la cual aquello que posibilita esa manifestación trascendente -sea la intencionalidad o la trascendencia- se exhibe de un modo inmanente. El problema no es ya el aparecer del objeto o ente, sino el aparecer del aparecer. Según Henry, el acto intencional o el movimiento de trascendencia hacia el mundo debe ser justificado a su vez por medio de una revelación que no les debe nada. Si no se nos revela o manifiesta, el acto intencional o el traspasamiento de la trascendencia carece de efectividad para nosotros, y por ende carece también de efectividad el horizonte del mundo dentro del cual se nos han de aparecer objetos o entes. Nos encontramos con una fenomenalidad originaria en el sentido de una revelación inmanente que es la condición ontológica de posibilidad de la manifestación de todos los fenómenos trascendentes. Este aparecer de la vida que se experiencia a sí misma es una autoafección o afectividad. No hace aparecer algo extraño ni se aparta de ella misma, es decir, no se relaciona sino consigo misma. Por eso tiene una significación material en el sentido de que se da a sí misma su propio contenido. Se trata de la fuente secreta de la fenomenalidad, esto es, el pathos no extático de la vida que se da independientemente de la fenomenalidad del mundo. Así, Henry distingue la reducción pura, en la que se oponen el contenido óntico y el aparecer que lo hace manifiesto, y la reducción radical a la inmanencia, en la que el poder que experiencia coincide con el contenido experienciado: "Prolongando la obra de la reducción pura y llevándola a su término, la reducción radical reduce el aparecer mismo, pone a un lado en él esta zona de luz que llamamos el mundo para descubrir aquello sin lo cual este horizonte de visibilización jamás llegaría a ser visible, a saber, la autoafección de su exterioridad trascendental en el pathos sin afuera de la Vida"2.

${ }^{1}$ Cfr. Michel Henry, L'essence de la manifestation, Paris, Presses Universitaires de France, 1963, Sec. I.

2 Michel Henry, Phénoménologie de la vie. I. De la phénoménologie, Paris, Presses Universitaires de France, 2004, p. 90. 
Henry señala que la vida trascendental del ego absoluto "no es otra cosa para nosotros que la vida misma de nuestro cuerpo originario"3. Poseemos respecto de este cuerpo un saber primordial en la autoafección porque el ejercicio de los poderes corporales es aprehendido inmediatamente sin ninguna distancia fenomenológica. El movimiento corporal nos es dado en una experiencia interna trascendental como una modalidad de la vida. En la actualidad de su ejercicio, el poder no se experiencia como exterior a sí mismo sino como el poder del "yo puedo" que soy. Del cuerpo fundado u orgánico como conjunto de poderes sobre el mundo es necesario diferenciar el cuerpo absoluto u originario como poder de ejercer esos poderes. Esta coincidencia del yo originario con el cuerpo originario exhibe una libertad originaria en la que soy colocado sin separación alguna al alcance de mis poderes en la inmanencia de la subjetividad.

Ahora bien, la vida del yo no es nunca el fundamento de su ser. Henry distingue un concepto débil y un concepto fuerte de autoafección. El sentido débil de autoafección tiene que ver con que tengo una experiencia de mí mismo sin ser la fuente de la experiencia. Me encuentro autoafectado, es decir, me soy dado a mí mismo sin que la donación proceda de mí. Según el concepto fuerte de autoafección que corresponde a la Vida absoluta, la Vida se autoafecta en un doble sentido. Por un lado, se autoafecta en el sentido de que ella misma define el contenido de su propia afección. Se da a sí misma el contenido que ella misma es. Por otro lado, se autoafecta en el sentido de que padece por sí misma el contenido de su afección. No pone fuera de sí el contenido como algo otro: "Este concepto fuerte de la autoafección es el de la vida fenomenológica absoluta y no es apropiado sino a ella, es decir, a Dios"4. Puesto que reposa sobre el advenimiento de la Vida a sí misma, el acceso del yo a sí mismo es vivido como esencialmente pasivo respecto de ese fenómeno primigenio. El yo se experiencia a sí mismo sobre el fondo de la Ipseidad originaria de la Vida que lo da a sí

${ }^{3}$ Michel Henry, Pbilosophie et phénoménologie du corps, Paris, Presses Univesitaires de France, 1965, p. 272.

${ }^{4}$ Michel Henry, C'est moi la verité. Pour une philosophie du cbristianisme, Paris, Éditions du Seuil, 1996, p. 135 (tr. cast.: Yo soy la verdad. Para una filosofia del cristianismo, Salamanca, Sígueme, 2001, p. 125). 
mismo y hace de él lo que es. El yo no se autoafecta sino que es autoafectado. Al experienciarse a sí mismo en la Ipseidad de la Vida, el yo entra en posesión de sí mismo y de cada uno de los poderes que lo atraviesan, y, al entrar en posesión de ellos, está en condiciones de ejercerlos. El yo tiene la capacidad de estar en posesión de sí y de ser uno consigo mismo y con todo lo que lleva en sí. Sin embargo, a cada uno de estos poderes se opone un no-poder. Se trata de la impotencia absoluta del yo respecto al hecho de hallarse en posesión de ese poder: "[...] porque separados de mí no podéis hacer nada" (Jn 15 5); “[...] pues Dios es quien obra en vosotros el querer y el obrar, como bien le parece [...]" (Flp 2 13). Considerar al ego como fuente u origen de los propios poderes, implica el olvido de la Vida que nos dona todos sus poderes.

Desde su primera obra publicada Henry toma como hilo conductor afirmaciones de Meister Eckhart: "El ser de Dios es mi vida. [...] Él me engendra como Él mismo y se engendra como mí mismo y me engendra como su ser y como su naturaleza"5. Puesto que el movimiento por el cual somos generados no es disociable del proceso eterno en que la Vida Absoluta adviene a sí misma, Eckhart sostiene que Dios se engendra como mí mismo. Puesto que la identidad de lo afectante y lo afectado no es puesta por el sí-mismo sino que la ipseidad resulta de la autoafección eterna de la Vida, Eckhart afirma que Dios me engendra como Él mismo.

\section{El estallido del fenómeno}

Según Heinrich Rombach, los fenómenos descritos por la fenomenología trascendental deben ser considerados -en un primer estallido del fenómeno- de un modo más profundo como epifenómenos de estructuras ontológicas fundamentales. Así, los fenómenos de conciencia aparecen como fenómenos derivados y nivelados respecto del ser-en-elmundo o el cuidado (Sorge). Además, estas estructuras tienen que ser remitidas a su vez -en un segundo estallido- a una historia fundamental del Dasein. Esto significa desarrollar una fenomenología estructural cuyo

${ }^{5}$ Meister Eckhart, Deutsche Predigten und Traktate (ed. Josef Quint), München, Carl Hanser, 1978, p. 184 s. Cfr. M. Henry, L'essence de la manifestation, pp. 385-418, $532-$ 549 ; y C'est moi la vérité, pp. 132 y ss. (tr. pp. 122 y ss.). 
análisis no parte de un núcleo fijo del hombre sino del modo histórico en que se genera una estructura o situación fundamental. El doble estallido del fenómeno revela el "nexo estructural" que exhiben diversas épocas o culturas, y en el cual los fenómenos más significativos asumen diferentes modos de expresión y se entretejen en una constelación total que constituye la esencia del hombre de ese momento histórico. Si bien mantiene la reducción como presupuesto, Rombach exige ir más allá de ella: "La 'reducción fenomenológica' es solo la puerta de entrada que debe ser atravesada, pero no debe significar ninguna fijación"6.

La fenomenología estructural es la teoría de la fuerza interior y oculta de autotrascendencia que opera constantemente y permite que la marcha total (Gesamtgang) de la naturaleza sea un avance emergente (Hervorgang) en virtud de una intencionalidad inherente a las cosas mismas. Estas se trascienden a sí mismas porque el todo está presente en ellas y permite que incluyan más que el contenido de su propio ser. El análisis intencional de Husserl es aplicado a un nuevo nivel de modo que no se trata ya de examinar las intenciones inherentes al sujeto sino aquellas que se encuentran en las cosas en el sentido de una tendencia al despliegue y la elevación. Este proceso es solidario con una concreatividad entre el hombre y la naturaleza o sus congéneres a través de una génesis de estructuras cuyo acontecer (Gescheben) es puro porque no presupone un portador, es decir, es más originario que el hombre, la naturaleza o el prójimo. Hay sucesos que no derivan de ninguna de estas instancias y que no pueden ser anticipados o planeados. En ellos lo uno se enlaza con lo otro en un acontecimiento del que emergen a la vez el hombre, la sociedad y la naturaleza. Rombach sostiene que en todo proceso de esta índole "nace un sentido concreto que no se puede determinar a partir de ninguna previa fijación de metas o definición

${ }^{6}$ Heinrich Rombach, Die Welt als lebendige Struktur, Freiburg i. Br., Rombach, 2003, p. 24. Cfr. pp. 51 y ss., y Heinrich Rombach, "Das Phänomen Phänomen", Phänomenologische Forschungen, 9, Freiburg/München, Karl Alber, 1980, pp. 11 y ss. "Así como la fenomenología solo llega a ser posible en cuanto tal por el concepto de estructura, el pensamiento estructural sólo llega a ser científicamente presentable en cuanto tal por el método fenomenológico" (Heinrich Rombach, Der Ursprung. Pbilosophie der Konkreativität von Mensch und Natur, Freiburg i. Br., Rombach, 1994, p. 179). 
de métodos"7. La realidad es creadora y pone algo a partir de sí misma de modo que el hombre puede aprovechar las fuerzas que ella aporta y obtener más de lo anticipado en sus proyectos. Lo importante no es efectivizar las propias representaciones en las cosas consideradas como un mero material, sino estimular su autoelevación induciendo en ellas una respuesta: "El hombre no es un lugar particular de la naturaleza, él es solo el lugar en el cual acontece en particular lo que en la naturaleza acontece en general, a saber, que las diferentes estructuraciones de la voluntad de vida de la naturaleza, tan solo por medio de su encuentro concreativo, son estimuladas y obligadas a la liberación de las fuerzas de autotraspasamiento (Selbstübersteigung), y de ese modo justamente producen un 'avance"'8.

$\mathrm{Al}$ efectuar el acontecer puro en nuestra vida individual, participamos en la realización del puro acontecer de nuestra especie y en el puro acontecer de la vida o el ser en cuanto tales. El acontecer puro no transcurre en una dimensión ontológica ya existente sino que crea una nueva dimensión ontológica mediante la génesis de una estructura que implica una transformación que puede asumir tres formas. En primer lugar, se presenta como el cambio (Umschlag) que da lugar a nuevas especies o géneros mediante una sucesión pacífica en el ámbito de la historia natural con la creación de nuevas formas, es decir, géneros y especies. En segundo lugar, aparece el trastocamiento (Umstury) que da lugar a épocas o culturas en el ámbito de la historia humana como productos de estados de tensión en una vieja forma. A la producción de especies en la naturaleza corresponde la organización de mundos de la vida en este nuevo nivel, pero no por sucesiones pacíficas como las de la naturaleza sino por el conflicto combativo entre épocas o culturas que desempeñan el papel de los géneros y las especies. Por último, la ruptura $(U m b r u c h)$ ha de dar lugar a la posthistoria no violenta y plural en que los diversos mundos se estiman en sus diferencias laterales, sin englobarese verticalmente en una totalidad ${ }^{9}$.

${ }^{7}$ Heinrich Rombach, Die Gegenwart der Pbilosophie, Freiburg/München, Karl Alber, 1988, p. 219.

8 Heinrich Rombach, Strukturantbropologie "Der menscbliche Menscb", Freiburg/München, Karl Alber, 1987, p. 128 (tr. cast.: El bombre bumanizado. Antropologia estructural, Barcelona, Herder, 2004, pp. 131 y ss.

${ }^{9}$ Cfr. ibid., pp. 84-94 (tr. pp. 86-97). 
El mundo es mediado por situaciones, y éstas se incluyen en él a través de diversos niveles. Varios círculos situacionales configuran la organización anular o radial de la llamada "escarapela de la situación". Cada anillo se asocia con la diferencia entre una situación exterior que concierne y una situación interior o yo que es concernido o afectado. De ahí que, en virtud de esta concernencia (Angang), los contenidos de la situación tienen un movimiento hacia un centro que se constituye como dativo ontológico del darse del mundo. Hay una movilidad concéntrica de la situación en la medida en que todo lo que le es inherente se acerca, concierne e incide en el yo. Una situación no es un ámbito exterior que rodea, sino que está asentada en lo más interior de cada uno de modo que el sí-mismo se experiencia como entregado a ella y disperso en ella. Las situaciones constituyen el símismo en tanto lo atañen o apuntan a él en su organización radial. Convergen en una consecuencia que es la situación de situaciones, y que no es otra instancia que el yo mismo en tanto vivencia de la consecuencia de las situaciones. El mundo se refleja en un sentir de tal modo que el estar dentro (Innensein) de la situación es a la vez un percatarse (Innesein) de ella. Lo cual significa que el yo no es una instancia previa para las situaciones sino la autoexperiencia de una multiplicidad de situaciones en la unidad de una concernencia. Y sólo se encuentra a sí mismo en un retorno a partir de la situación, esto es, como una contramarcha (Gegengang) en respuesta a la concernencia. Puesto que toda situación me concierne y esto implica un "a mí" ulterior, no se puede pensar en una situación más interna caracterizable en sí misma, y, por tanto, se debe reconocer un abismo o nada interior.

Los círculos situacionales se extienden desde el círculo circunscrito por el yo y el nosotros, pasando por la situación de culturas y épocas, hasta los círculos vitales de la naturaleza y el círculo más externo del todo de la vida (All-Leben), esto es, lo omniabarcador que no es una situación singular sino la fuente de todas las situaciones y del avance emergente de la realidad. Este origen se encuentra tanto en los márgenes como en cada punto de la realidad a través de una refracción o espejamiento. Tanto el todo de la vida como las situaciones naturales más lejanas solo pueden ser captadas a través de las situaciones que resultan de culturas y épocas, esto es, aquellas situaciones intermedias cuya función es mediatizar las situaciones individuales de cercanía con las situaciones más amplias y lejanas. Así, el todo de la vida adquiere una forma concreta a través de mitos, cosmogonías, 
religiones y filosofías. Sin embargo, "el conjunto del acontecer cósmico" o "el ser más elevado, superior y más abarcador"10 no puede ser fijado o convertido en tema, ni nombrado porque no es una situación determinada. Los nombres que ha recibido -Brahma, Apeiron, Ser, Dios, Realidad, etc.no son adecuados y por eso se la ha llamado lo "sin nombre". Rombach recuerda una afirmación de Eckhart: "Está libre de todos los nombres y desprovisto de todas las formas, completamente solo y libre, [...]"11. A fin de no ser pensado en forma mundana, necesita de un sentido propio e inderivable que sólo se puede entender a partir de su propia condición, y que, por tanto, tiene el carácter del "por-sí-mismo" (Von-selbst). Aquello que trasciende lo finito no es algo que existe ante nosotros sino lo que acontece "si este acontecer -aclara Rombach- no es pensado como apéndice de lo existente, sino como su origen, como 'avance emergente', como 'acontecer puro"'12. Dios es pensado a partir de sí mismo como fuente de todas las situaciones.

El hombre debe ser liberado para un sí-mismo esencialmente superior, y este sí-mismo sólo se anuncia en la apertura a la situación más externa que, al igual que el abismo interior, debe ser considerada como una nada porque no es caracterizable como esto o aquello: "La teoría de la nada converge con la teoría del verdadero si-mismo, y las dos cosas se enlazan mediante el 'vacío' que las plenifica"13. Lo más abarcador no puede ser nombrado, pero puede ser experienciado. Esta vivencia del todo de la vida como la situación más externa equivale a la elevación del yo a la condición de yo total $(A l l-I c b)$. Aquellas situaciones en que lo más externo se hace presente evocan el verdadero yo que se encuentra olvidado y que no es otro que ese abismo interior al que remite todo darse de una situación: "El abismo más interior y el entorno más exterior son uno y el mismo"14.

$\mathrm{El}$ acontecer puro constituye el tema de una hermética que no se presenta como un saber secreto sino como "el llegar a ser patente del puro

\footnotetext{
${ }^{10}$ H. Rombach, Strukturanthropologie, p. 279 (tr. p. 285).

${ }^{11}$ M. Eckhart, op. cit., p. 163. Cfr. H. Rombach, Strukturanthropologie, p. 311 (tr. p. 317).

${ }^{12}$ H. Rombach, Strukturanthropologie, p. 280 (tr. p. 286).

13 Ibid., p. 313 (tr. p. 320).

14 Ibid., p. 318 (tr. p. 325).
} 
avance emergente"15. Es la captación y exposición del nacimiento de sentido y del alumbramiento de la realidad en cuanto tal. Un mundo o una estructura sólo es tal en su emerger, y la experiencia hermética capta este momento de irrupción, de acrecentamiento y mejoramiento, es decir, del simple "más" inherente al avance creador. En conexión con los tres modos de fenomenología -trascendental, hermenéutico, y estructural-, Rombach distingue, en una división de trabajo sin conflictos, las respectivas tres formas de realidad -cosa, texto e imagen-, de percepción -intuición, comprensión y visión-, de teoría -teoría del conocimiento, hermenéutica y hermética-, y de nexo -nexo de cosas, nexo de sentido y mundo-. En la hermética, el mundo que emerge es captado en una poesía o una obra de arte mediante una imagen que lo contiene en una unidad cerrada sin diferenciación y que puede ser vista en configuraciones mundanas concretas $^{16}$.

\section{El fenómeno saturado}

Jean-Luc Marion alude a una tercera reducción más allá de la reducción trascendental husserliana y de la reducción heideggeriana del ente al ser. Se trata de una reducción ordenada a lo puramente dado y a su darse, es decir, una reducción a la donación: "Sólo una fenomenología de la donación puede volver a las cosas mismas, porque para volver a ellas es necesario primero verlas, verlas venir $y$, en una palabra, sostener su arribo"17. Así como la objetividad sólo puede aparecer en su lazo con el objeto, y el ser en su diferencia respecto del ente, la donación sólo puede aparecer indirectamente y ser leída en la superficie y en el pliegue de lo dado.

${ }^{15}$ Heinrich Rombach, Der kommende Gott. Hermetik - eine neue Weltsicht, Freiburg i. Br., Rombach, 1991, p. 123.

16 " $\mathrm{Si}$ algo es visto como imagen o convertido en imagen, entonces el todo o lo absoluto llega a la presencia en un acontecer completamente concreto, en un lugar completamente concreto, en una forma completamente simple y casual" ( $\mathrm{H}$. Rombach, Der kommende Gott, p. 129). Por ejemplo, la atmósfera aparece en un cuadro de Turner como imagen del todo porque no se muestra en el paisaje o en los objetos sino que éstos se presentan en ella.

17 Jean-Luc Marion, Étant donné. Essai d'une phénoménologie de la donation, Paris, Presses Universitaires de France, 1997, p. 9. 
Ella aparece de contragolpe cuando se pone en escena el fenómeno en tanto pura y estrictamente dado sin privilegiar los modelos del ente y el objeto. Por ejemplo, el cuadro se reduce tanto más a su donación cuanto más se pone entre paréntesis su condición de ente o de objeto. Más allá de lo que se muestra de esta manera, un cuadro da su efecto. Por eso la reducción a la donación es la reducción al efecto invisible que el cuadro hace visible cuando se separa de lo que lo soporta. Su aparecer pretende darse como pura superficie sin soporte, o como una pura superficie que absorbe el soporte: "El cuadro [...] se reduce a su fenomenalidad última en tanto da su efecto"18.

El avance sobre la reducción trascendental y la reducción ontológica evoca la reducción, propuesta por Lévinas, de lo dicho al Decir, y Marion sostiene que "todos nos hemos convertido en levinasianos, y definitivamente"19. Lévinas interpreta la reducción trascendental como reducción de lo otro a lo mismo ligado a la propia subjetividad. Frente a ello, la irrupción del prójimo en tanto rostro significa el fin de la dominación de lo otro por lo mismo en favor de una ética como teoría del respeto a lo absolutamente otro y a una noción de sentido previa a toda donación de sentido por un sujeto $^{20}$. Marion retoma la idea de que el rostro se

${ }^{18}$ Ibid., p. 76.

19 Jean-Luc Marion, "La voix sans nom. Hommage - à partir - de Lévinas", Rue Descartes, N 19, 1998, p. 12.

${ }^{20}$ Cfr. Emmanuel Lévinas, Totalité et infini. Essai sur l'exteriorité, Phaenomenologica 8, La Haye, Martinus Nijhoff, 1971, p. 22. Lévinas contrapone a la reducción trascendental, en virtud de "la demasia (surplus) de la responsabilidad" inherente al para el otro, "la significancia de una significación que va más allá de lo dado y que se distingue de la famosa Sinngebung o 'donación de sentido"' (Emmanuel Lévinas, Dieu, la morte et le temps, Paris, Grasset, 1993, p. 202). Negativamente, el rostro no es un objeto o un ente sino que escapa a la intencionalidad husserliana y la trascendencia heideggeriana. Positivamente, es una voz que me llama a la responsabilidad por el otro de modo que no guardo nada para mí y respondo de un modo infinito. Por tanto, al decir como donación de sentido o noesis, que tiene su correlato en un significado dicho en tanto noema, antecede un Decir como responsabilidad que excede lo dicho: "Antes de colocarse al servicio de la vida como intercambio de informaciones a través de un sistema lingüístico, el Decir es testimonio, Decir sin Dicho, signo dado al Otro" (Emmanuel Lévinas, Autrement 
fenomenaliza porque se da en el modo de la interpelación: “[...] el rostro se da como una interpelación y no se muestra (no se fenomenaliza) sino en la medida en que el adonado responde"21. Además, Marion generaliza la cuestión de modo que una fenomenalización ajena a la objetividad y la entidad no se limita al rostro ni al horizonte de la ética, sino que es inherente a múltiples fenómenos que requieren una respuesta a lo que se da, es decir, una responsabilidad. El adonado que responde pierde el rango trascendental junto con la espontaneidad y la actividad que es inherente a esta condición, pero no cae en una pasividad, sino que asume una receptividad a la vez activa y pasiva que implica la capacidad de acrecentarse a la medida de lo dado a fin de sostener su arribo.

Marion presenta una tópica de diferentes tipos de fenómenos. El hilo conductor es el grado de intuición. En primer lugar, se encuentran los fenómenos pobres de intuición en que la intención significativa puede anticipar la intuición. Además, la intuición no es la intuición real de lo individual sino la intuición formal o categorial de objetos matemáticos o lógicos y la intuición generalizadora pura de esencias. Estos objetos tienen el privilegio de la certeza, pero exhiben un déficit fenomenológico ya que se sustraen a la individualidad y la temporalidad. En segundo lugar, se encuentran los fenómenos de derecho común en que, si bien la intención significativa también puede prever su impleción, la intuición plenificadora es más rica porque es una intuición de lo individual. Son los objetos del mundo percibido, los objetos de las ciencias de la naturaleza y los objetos de la técnica. Por último, se encuentran los fenómenos saturados en que la intuición se acrecienta al punto en que sobrepasa la intención significativa

qu'être ou au-delà de l'essence, Phaenomenologica 54, La Haye, Martinus Nijhoff, 1974, p. 192). En tanto significancia de la responsabilidad por el otro y la exposición al otro, el Decir se desfigura al mostrarse en algo dicho, y por eso es necesario reducir o desdecir lo dicho para encontrar el Decir ya no por medio de la identificación y la tematización atada a la esfera del ser y la esencia sino por medio de testimonio del uno-para-el-otro: "El ascenso hacia el Decir es la Reducción fenomenológica en que se describe lo indescriptible" (ibid., p. 69). Lévinas se refiere a "la reducción de lo Dicho al Decir, más allá del Logos, del ser y del no ser - más allá de la esencia - de lo verdadero y lo no verdadero - la reducción a la significación, al uno-para-el-otro de la responsabilidad" (ibid., p. 58).

${ }^{21}$ J.-L. Marion, Étant donné, p. 404. 
que no puede anticiparla. El fenómeno saturado tiene su propia tópica cuyo hilo conductor ya no es el grado de intuición, porque siempre hay saturación, sino la determinación respecto de la cual se realiza la saturación, es decir, la cantidad, la cualidad, la relación y la modalidad. Siguiendo el hilo conductor de las categorías kantianas, Marion distingue cuatro tipos de fenómenos saturados, es decir, paradojas que no pueden constituirse como objetos de acuerdo con un horizonte de anticipación: "El fenómeno saturado se describirá como inmentable (invisable) según la cantidad, insoportable según la cualidad, absoluto según la relación, incontemplable según la modalidad"22.

El primer tipo de fenómeno saturado, en el que se satura la categoría de la cantidad, es el acontecimiento histórico. Puesto que excluye un "aquí y ahora" respecto del cual podría ser descrito en forma exhaustiva, es posible adoptar sobre un suceso de esta índole un número infinito de perspectivas. De modo que se revela algo que, desde una pluralidad de horizontes, siempre hay que reconstituir. Así, el conocimiento del acontecimiento histórico es intersubjetivo e histórico, se realiza apelando a múltiples ciencias, y tiene el carácter de una hermenéutica inacabable. Por tanto, en tanto queda entregado a una teleología sin fin de carácter intersubjetivo e histórico, se asocia con una interobjetividad. Marion ve el caso ejemplar de un análisis fenomenológico de este tipo de fenómeno saturado en el tercer tomo de Temps et récit de Paul Ricoeur ${ }^{23}$.

La categoría de cualidad es subvertida por el ídolo, es decir, lo visible cuyo esplendor bloquea la intencionalidad como un obstáculo. Su caso privilegiado es el cuadro. La intuición sobrepasa siempre los conceptos que se proponen para dar cuenta del cuadro, y no basta una mirada para tener una verdadera visión. Así, el cuadro encubre siempre lo esencial de su visibilidad. Por medio de conceptos o informaciones es posible alcanzar una comprensión cada vez mayor de lo dado del cuadro, pero entonces la donación misma llega a ser inaccesible: "Lo dado intuitivo de ídolo nos impone cambiar sin cesar de mirada, aunque sólo sea para afrontar el insoportable deslumbramiento" 24 . El ídolo se distingue del acontecimiento

\footnotetext{
22 Ibid., p. 280.

${ }^{23}$ Cfr. ibid., p. 319 s.

${ }^{24}$ Ibid., p. 321.
} 
histórico porque no presupone una interobjetividad y una comunicación teleológica, sino que da lugar a un inevitable solipsismo al ejercer una individuación sobre la mirada que le hace frente.

El tercer caso de fenómeno saturado es el cuerpo propio o carne (chair) que se sustrae a la categoría de la relación. Ha sido analizado por Henry a través de la fenomenalidad inherente a la autoafección que se realiza sin condición, es decir, sin relación con algo externo. Este fenómeno presenta dos características singulares. Por un lado, a diferencia del ídolo, no puede contemplarse porque la inmediatez de la autoafección impide el espacio en que sería posible la separación extática inherente a la intencionalidad. Por otro lado, en contraste con el acontecimiento histórico, la carne provoca el solipsisimo porque la autoafección es intrínsecamente individual.

Por último, liberado de las categorías de la modalidad que no determinan los objetos en sí mismos sino su relación con el yo trascendental, se encuentra el tipo de fenómeno no saturado que corresponde al rostro de Lévinas y que Marion denomina "ícono". Se trata de un fenómeno que ya no ofrece un espectáculo a la mirada ni tolera la mirada de un espectador sino que ejerce su propia mirada convirtiendo al contemplante en contemplado. Así, el fenómeno manifestado se convierte en una automanifestación a partir de sí, es decir, toma la iniciativa de la manifestación. Es el rostro del otro que no nos da nada para ver sino que pesa sobre nosotros. El ícono encierra en sí los caracteres particulares de los tres tipos precedentes de fenómenos saturados. Al igual que el acontecimiento histórico, exige una suma de horizontes y narraciones porque da lugar a una teleología. Del mismo modo que el ídolo, el ícono exige una reiteración de la visión e individualiza la mirada. $\mathrm{Y}$ al igual que la carne, realiza esta individuación afectando tan originariamente al yo que este pierde su función de polo trascendental. Así, el ícono reúne en sí todos los modos de saturación y, por tanto, muestra que, en el interior de la región privilegiada de la saturación, los fenómenos no ofrecen el mismo grado de donación.

Por tanto, si hay una gradación en la donación, se impone la cuestión de determinar hasta qué punto puede desplegarse la saturación. El máximo se encuentra en el fenómeno de la Revelación que concentra en sí los cuatro 
tipos de fenómenos saturados porque se da como acontecimiento histórico, ídolo, carne e ícono o rostro en una saturación de quinto tipo, "no porque ella añada un tipo de nuevo (arbitrariamente inventado para hacer justicia al derecho supuesto de lo 'divino') a los cuatro precedentes (los únicos descriptibles), sino porque confundiéndolos en él, satura la fenomenalidad en el segundo grado, por saturación de saturación"25. Marion describe el fenómeno de la revelación como una simple posibilidad sin presuponer su efectividad -esto corresponde a la teología revelada- y a la vez proponiendo una figura precisa. Una revelación efectiva sólo es posible si se da según el tipo de paradoja por excelencia que se ha descrito. $Y$ subraya que el fenómeno del Cristo se da intuitivamente como un acontecimiento imprevisible porque es heterogéneo a lo que sin embargo da cumplimiento, es decir, las profecías. Hay una saturación según el acontecimiento imprevisible en tanto su "hora" escapa al tiempo del mundo y a toda preparación en el pasado. La actitud apropiada frente al acontecimiento queda definida por la espera sin previsión: "Velad pues porque no sabéis qué día vendrá nuestro Señor" (Mt 24 42). Según la cualidad, la figura del Cristo supera lo que la mirada fenomenológica puede soportar. Hay aquí una saturación según lo insoportable porque su figura escapa al espacio de la tierra: "Mucho tengo todavía que deciros, pero ahora no podéis con ello" (Jn 16 12). Según la relación, el Cristo satura todo horizonte en el que pudiera introducirse una relación porque "mi Reino no es de aquí" (In 18 36). Según la modalidad, el Cristo aparece en tanto fenómeno incontemplable precisamente porque a título de ícono me contempla de tal manera que soy constituido por él como testigo, y no él por un yo trascendental. A esta inversión de la mirada se refiere la afirmación de que “el siervo no es más que su señor" (Jn 1520 ).

\section{La excedencia}

Las tres posiciones examinadas se vinculan entre sí no solo por la consideración de un tercer nivel de la fenomenología -más allá de los asociados con Husserl y Heidegger-, sino también por una reflexión que, en el terreno del tercer nivel, gira en torno de la dualidad entre lo que aparece y

25 Ibid., p. 327. 
el aparecer y descubre una excedencia en relación con algún aspecto de ella. En un ahondamiento del aparecer, la excedencia recae en la inmanencia de la vida que, como característica del aparecer en cuanto tal, se encuentra más allá del mundo. En una profundización de lo que aparece, y como una condición general, es asignada a nuevos mundos que emergen como resultado de un acontecer puro. $Y$ en una explicitación del grado de impleción en la relación entre lo que aparece y el aparecer, se presenta en ciertos fenómenos particulares que se dan en el mundo y que son preeminentes en razón de la saturación que contienen.

Ante la dualidad de lo que aparece y su aparecer, Henry destaca que el aparecer debe aparecer en cuanto tal porque de lo contrario nada puede aparecer. Insiste en que es absurdo considerar que pueda haber revelación del mundo sin revelación inmanente. Además, subraya que, cuando se experiencia a sí mismo, el viviente experiencia la Vida absoluta que a su vez se experiencia en él. El viviente está desbordado por una trascendencia que está presente en toda modalidad inmanente de la vida: “Así, es necesario llevar la reducción a su término, según el 'más' que la radicaliza para que la donación se dé a sí misma según su propio exceso, [...]"26.

Según Rombach, la dualidad de lo que aparece y su aparecer es interpretada por Husserl de tal modo que ambos términos se colocan dentro del fenómeno. Esto significa situar en el fenómeno no solo el horizonte de la posible objetividad sino también la plena materialidad del objeto efectivo. Husserl esquiva el emerger de la realidad mediante esta distinción interna, pero ese emerger tiene como consecuencia un estallido del fenómeno en dirección a una dimensión ontológica e histórica permanentemente renovada por un "más" que da lugar a nuevos mundos experienciados ${ }^{27}$. Tal experiencia implica emerger hacia un sujeto más amplio, y este surgimiento produce una transformación del propio sí-mismo en virtud de una elevación o intensificación de la ipseidad. No se produce una generalización del sujeto sino una mayor intimidad e intensidad junto con un acrecentamiento y mejoramiento: "Esto significa que el 'objeto' de la experiencia hermética no

${ }^{26}$ M. Henry, Phénoménologie de la vie I, p. 91.

${ }^{27}$ Cfr. H. Rombach, "Das Phänomen Phänomen", pp. 12 y ss. 
aparece, por ejemplo, como más cercano o más certero o más preciso sino como 'más"'28.

Para Marion, la caracterización husserliana del fenómeno a través de la dualidad del aparecer y lo que aparece se despliega según el par significado/impleción o intención/intuición que admite una relación entre los términos en juego tal que la intuición puede exhibir una excedencia desmesurada. Marion propone pensar el fenómeno de derecho común y, a través de él, el fenómeno pobre en intuición a partir del paradigma del fenómeno saturado. En lugar de considerar la paradoja como un caso excepcional de la fenomenalidad, subraya que solo los fenómenos saturados permite ilustrar el "único paradigma de la fenomenalidad" 29 de modo que los fenómenos de derecho común y los fenómenos pobres en intuición son variantes debilitadas que derivan por un proceso de extenuación progresiva: "En esta hipótesis, la imposibilidad de alcanzar un conocimiento del objeto, una comprensión en sentido estricto, no proviene de la falla de la intuición dadora, sino de su acrecentamiento (surcrô̂t) que ningún concepto, ni significación, ni intención puede prever, organizar o contener" 30 .

Los temas fundamentales han sido la radicalización de la reducción, una dimensión preobjetiva de la experiencia, el fenómeno de la excedencia y la fuente divina de esta excedencia. De todos ellos se encuentra una anticipación en la fenomenología trascendental. Ante todo, Husserl introduce la exigencia de ir más allá de la reducción del mundo al curso de la conciencia al reconducir este flujo a su presente viviente y permanente mediante "la reducción radical" 31 . Además, ofrece un análisis egológico en profundidad en que el curso de vida del yo, si bien es bilateral porque contiene una protohyle como momento extraño, exhibe una unidad inseparable en la que, si se los considera por separado, esos momentos son

${ }^{28}$ H. Rombach, Der kommende Gott, p. 48.

${ }^{29} \mathrm{~J}$.-L. Marion, Étant donné, p. 316.

30 Jean-Luc Marion, De surcroît. Études sur les phénomènes saturés, Paris, Presses Universitaires de France, 2001, p. 192.

31 Hua XXXIV, 185. La sigla corresponde con indicación de tomo y página a Edmund Husserl, Husserliana-Gesammelte Werke, vols. I-XXXIX, y-Materialien, vols. I-VIII, Dordrecht, Springer (con anterioridad: Haag, Martinus Nijhoff; Dordrecht/Boston/London, Kluwer Academic Publishers), 1950-2007. 
abstractos. Por tanto, la intencionalidad en tanto "conciencia de" es "un resultado constitutivo"32. Asimismo, la excedencia es intrínseca a la intencionalidad a través de sus horizontes abiertos, indeterminados e inagotables. Esto significa que, para Husserl, el fenómeno de derecho común es saturado en el sentido de que excede a la anticipación significativa que siempre se desenvuelve en un nivel de generalidad y pobreza respecto de la intuición, y que los fenómenos pobres en intuición también son saturados porque, en tanto objetos ideales, tienen sus horizontes ${ }^{33}$. Por último, la fenomenología de Husserl culmina en una teleología y teología filosófica. Si la constitución del mundo es solo un hecho casual y perecedero que puede terminar en un caos, la vida humana queda afectada por una carencia de sentido, y -siguiendo a Kant- se debe postular un ser trascendente que garantice el sentido de la vida: "Pero si creo y hago consciente esta creencia, la efectúo libremente a partir de esta fuente práctica, entonces ella da sentido al mundo y a mi vida, proporciona la regocijada confianza (die freudige Zuversicht) en que nada es en vano y todo es para el bien"34. En un manuscrito, Husserl se expresa en estos términos: "Para creer en mí mismo y en mi verdadero yo, y en el desarrollo en esta dirección, debo creer en Dios, y en la medida en que hago esto, veo la guía divina, el consejo de Dios, la exhortación divina en mi vida" 35 .

\section{Resumen}

Luego de Husserl y Heidegger, la fenomenología ha intentado llevar la reducción más allá de la referencia de los objetos a las operaciones efectuadas por la conciencia o de los entes al ser. En primer lugar, un nuevo nivel de reducción se manifiesta en la reducción radical propuesta por M. Henry para retroceder del aparecer al aparecer del aparecer, y conduce al descubrimiento de una dimensión en que no hay horizontes que puedan ser plenificados porque impera la sobreabundancia del la vida. En segundo lugar, según $\mathrm{H}$. Rombach, los fenómenos descritos en los dos primeros niveles no son otra cosa que epifenómenos y tienen

32 HuaMat VIII, 335 n. Cfr. pp. 110, 199, 326 ss., 351 ss., 362.

${ }^{33}$ Cfr. Hua III/1, 287 s, Hua VIII, 309.

${ }^{34}$ Hua VIII, p. 355.

${ }^{35}$ Edmund Husserl, Manuscrito A V 21, 24 b. Citado según Ulrich Melle, "Husserl's Personalist Ethics", Husserl Studies, Vol. 23, No 1, 2007, p. 15. 
que ser explicados en términos de estructuras que emergen en un desarrollo creador. La realidad produce algo a partir de sí misma, y esta contribución debe ser aprovechada a fin de alcanzar más de lo anticipado en nuestros proyectos. En tercer lugar, J.-L. Marion presenta una teoría sobre los fenómenos saturados a fin de dar cuenta de aquellos casos que exceden los límites que les han sido delineados en las intenciones significativas vacías porque muestran una demasía de intuición. Las tres tendencias conducen respectivamente a una fenomenalidad originaria, a un estallido del fenómeno y a una saturación del fenómeno. El artículo sostiene que tienden a converger porque vinculan el tercer nivel de la fenomenología con una reflexión en torno de la dualidad entre lo que aparece y su aparecer, y que, al hacerlo, ponen de manifiesto una excedencia respecto de tres aspectos distintos de la dualidad, esto es, el aparecer en cuanto tal, la condición general de lo que aparece o la aparición de algunos fenómenos preeminentes. Finalmente, se indican algunas anticipaciones de estas tendencias en la fenomenología trascendental de Husserl.

Palabras clave: fenomenalidad, vida, estructura, saturación, excedencia.

\begin{abstract}
After Husserl and Heidegger, phenomenology has attempted to push the reduction beyond the reference of objects to the performances effected by consciousness, or of beings to Being. First, a new level of the reduction comes forth in M. Henry's radical reduction of appearing to the appearing of appearing, and leads to the disclosure of a dimension in which no horizons are to be fulfilled because the superabundance of life holds sway. Secondly, according to $\mathrm{H}$. Rombach, the phenomena decribed in the first two levels are nothing but epiphenomena, and must be explained in terms of structures that emerge in a creative development. Reality brings forth something from itself, and we must take advantage of this contribution in order to obtain more than what is anticipated in our projects. Thirdly, J.-L. Marion advances a theory on saturated phenomena in order to account for phenomena that surpass the bounds outlined for them in the mere significational intentions because they show a surplus of intuition. The three trends lead respectively to an originary phenomenality, an outbreak of phenomena, and saturated phenomena. The article contends that they tend to come together because they link the third level of phenomenology to a reflection that turns on the duality between what appears and its appearing, and, in doing so, disclose a surplus with regard to three different aspects of the duality, i.e., appearing as such, the overall condition of what appears, or the appearing of some preeminent
\end{abstract}


phenomena. Finally, some anticipations of these trends in Husserl's transcendental phenomenology are shown.

Key words: phenomenality, life, structure, saturation, surplus. 